\title{
Avaliação de hábitos alimentares de universitários em Paripiranga-BA
}

\author{
Evaluation of eating habits of university students in Paripiranga-BA
}

\section{Evaluación de hábitos alimentarios de universitarios en Paripiranga-BA}

Wellington Pereira Rodrigues ${ }^{1 *}$, Priscila Dantas Gonçalves ${ }^{2}$, Josefa Juliana de Oliveira ${ }^{2}$, Qhetillin da Silva Santos ${ }^{2}$, Robenildo dos Santos Brito², Fabíola Ferreira de Sousa ${ }^{2}$, Daniela de Souza ${ }^{2}$, Ana Paula Gomes Soares ${ }^{3}$, Renan Sallazar Ferreira Pereira ${ }^{4}$, Ingrid Borges Siqueira ${ }^{5}$.

\section{RESUMO}

Objetivo: O objetivo desse estudo foi avaliar hábitos alimentares e estilo de vida de adultos estudantes do Centro Universitário AGES. Métodos: Trata-se de estudo transversal descritivo, de campo, conduzido por 161 indivíduos, de ambos os sexos, idade de 20 a 59 anos. Os pacientes foram avaliados de acordo com a definição de alimentos funcionais. A restrição alimentar destes foi analisada por um questionário de frequência alimentar, que foi readaptado para alguns alimentos, de acordo com as categorias de consumo: habitual, não habitual, raramente consumido e nunca consumido. O estudo foi aprovado por Comitê de Ética em Pesquisa sob o parecer ํㅜ 024-2017. Resultados: Cerca de 43\% dos participantes da amostra que declararam consumir refrigerante, consomem mais de um litro por dia, causando riscos à saúde. Apesar dos resultados negativos já citados, o estudo aponta que há preocupação dos estudantes na qualidade dos hábitos alimentares, quando $45 \%$ destes alegam que preferem sucos naturais, $44 \%$ dos universitários relataram que só tomam refrigerante raramente e $53 \%$ responderam que leem os rótulos dos alimentos. Conclusão: Conclui-se que foi possível perceber que o consumo de industrializados de forma geral ainda é crescente, principalmente no público adulto e universitário, fazendo-se necessário a criticidade durante a compra do produto, bem como no momento das escolhas alimentares.

Palavras-Chave: Alimentos Funcionais, Dieta, Doença Crônica.

\begin{abstract}
Objective: The objective of this study was to evaluate eating habits and lifestyle of adult students of Centro Universitário AGES. Methods: This is a descriptive cross-sectional field study conducted by 161 individuals of both sexes, aged from 20 to 59 years. Patients were evaluated according to the definition of functional foods. The food restriction was analyzed by a food frequency questionnaire, which was readapted for some foods according to consumption categories: usual, not habitual, rarely consumed and never consumed. The study was approved by the Research Ethics Committee under opinion o 024-2017. Results: About $43 \%$ of participants in the sample who reported consuming soda consume more than one liter per day, causing health risks. Despite the negative results already cited, the study points out that students are concerned about the quality of eating habits, when $45 \%$ of them say they prefer natural juices, $44 \%$ of university students report that they only drink soda rarely and $53 \%$ say they read the labels of foods. Conclusion: It was concluded that it was possible to perceive that the consumption of industrialized products in general is still increasing, mainly in the adult and university public, making necessary the criticality during the purchase of the product, as well as in the moment of the food choices.
\end{abstract}

Key words: Functional Foods, Diet, Chronic Disease.

\footnotetext{
${ }^{1}$ Universidade Federal de Sergipe (UFS), Aracaju-SE. *E-mail: wellington_life@live.com

2 Centro Universitário AGES (UNIAGES), Paripiranga-BA.

3 Universidad Federal de Viçosa, Minas Gerais - MG

4 Universidad Federal do Tocantins (UFT), Tocantins-MS

5 Universidad Tiradentes (Unit), Aracaju-SE.
} 


\section{RESUMEN}

Objetivo: El objetivo de este estudio fue evaluar hábitos alimenticios y estilo de vida de adultos estudiantes del Centro Universitario AGES. Métodos: Se trata de un estudio transversal descriptivo, de campo, conducido por 161 individuos, de ambos sexos, edad de 20 a 59 años. Los pacientes fueron evaluados de acuerdo con la definición de alimentos funcionales. La restricción alimentaria de estos fue analizada por un cuestionario de frecuencia alimentaria, que fue readaptado para algunos alimentos, de acuerdo con las categorías de consumo: habitual, no habitual, raramente consumido y nunca consumido. El estudio fue aprobado por el Comité de Ética en Investigación bajo el dictamen n ${ }^{\circ}$ 024-2017. Resultados: Cerca del $43 \%$ de los participantes de la muestra que declararon consumir refrigerante, consumen más de un litro al día, causando riesgos para la salud. A pesar de los resultados negativos ya citados, el estudio apunta que hay preocupación de los estudiantes en la calidad de los hábitos alimentarios, cuando el $45 \%$ de éstos alegan que prefieren jugos naturales, el $44 \%$ de los universitarios relataron que sólo toman refrigerante raramente y el $53 \%$ respondieron que leen las etiquetas de los " los alimentos. Conclusión: Se concluye que fue posible percibir que el consumo de industrializados de forma general sigue siendo creciente, principalmente en el público adulto y universitario, haciéndose necesario la criticidad durante la compra del producto, así como en el momento de las elecciones alimentarias.

Palabras clave: Alimentos Funcionales, Dieta, Enfermedad Crónica.

\section{INTRODUÇÃO}

A alta prevalência de obesidade no Brasil e o agravo das doenças Crônicas não transmissíveis (DCNTs) como diabetes, hipertensão e doenças cardiovasculares são a primeira causa de morte no mundo, além de serem diretamente relacionadas com a alimentação desregulada. Estudos apontam que uma alimentação de qualidade pode agir com caráter preventivo nas DCNTs, bem como em outras patologias não necessariamente crônicas, como dislipidemias e sobrepeso (FERREIRA, 2010; ORLANDO et al, 2010).

Contudo, percebe-se que grande parte da população ainda não dá a importância necessária a alimentação equilibrada, nem tão pouco aos altos índices de morbidade e mortalidade causados por uma alimentação inadequada. Atualmente, o público de adolescentes e adultos são os mais afetados pela rotina acelerada e, consequentemente, pela má alimentação, que pode desencadear consequências negativas a curto e longo prazo (BARRETO ML, 2013; OLIVEIRA JS, et al., 2017).

Doenças como o diabetes, por exemplo, encontram-se entre as 10 primeiras causas de morte em vários países. Causas essas que são de elevada prevalência, bem como seus fatores de risco. Dentre esses fatores, destacam-se o tabagismo, o consumo abusivo de bebidas alcoólicas, mas sobretudo a obesidade e 0 consumo excessivo de gorduras saturadas que desencadeiam às dislipidemias (ENES CC, 2010). Neste sentido, os bons hábitos alimentares podem ser estratégias eficazes para prevenção, controle e auxílio no tratamento de patologias relacionadas. A ingestão insuficiente de frutas e hortaliças e a inatividade física são fatores alarmantes no país atualmente, e contribuem para o aumento das morbidades associadas ao sedentarismo e má alimentação. Dados do Brasil (2017) apontam que o consumo de hortaliças e frutas aumentou, mas não em quantidade suficiente, bem como os exercícios físicos.

O objetivo desse estudo foi avaliar hábitos alimentares e estilo de vida de adultos estudantes do Centro Universitário AGES. A investigação é de relevância social e profissional, uma vez que traz um retrato da atual situação dos hábitos alimentares desse público, traçando assim um perfil desses sujeitos no contexto da nutrição na sociedade atual, possibilitando uma interferência nessa realidade situacional. A hipótese deste estudo é que maior parte dos indivíduos não se alimente de forma saudável, o que vai de encontro com maioria dos estudos publicados atualmente.

\section{MÉTODOS}

O presente estudo foi realizado com acadêmicos do Centro Universitário AGES, localizados no município de Paripiranga, Bahia, sem seleção de curso. Os mesmos estavam com faixa etária de 18 a 59 anos. Os critérios de exclusão foram apenas para adolescentes e idosos e pessoas que não estudam na instituição de ensino. Os critérios de inclusão foram: adultos e estudantes do ensino superior na UniAGES. 
Os mesmos teriam que responder a um questionário com 8 perguntas, que se referiam a consumo de refrigerantes e outros alimentos industrializados, bem como sua frequência semanal, consumo de bebidas industrializadas, leitura de rótulos e compreensão acerca disso. O questionário foi validado através de prétestes, aplicados em pessoas próximas aos autores.

Os indivíduos foram abordados nas praças de alimentação da instituição por alunos do $6^{\circ}$ período do curso de nutrição. A análise de dados foi realizada através de tabulação no software Microsoft Excel 2013, no qual calculou-se a média, desvio padrão, frequência relativa e absoluta dos dados obtidos. Para a revisão de literatura foram priorizados artigos atuais, do ano de 2010 a 2017. O estudo foi aprovado pelo Comitê de Ética e Pesquisa com Seres Humanos do Centro Universitário AGES sob o parecer n 024-2017.

\section{RESULTADOS e DISCUSSÃO}

Dos 100 entrevistados no do Centro Universitário AGES, 64\% são do gênero feminino, já a idade dos entrevistados possui média de 24,42 (Tabela 1).

Tabela 1 - Média e desvio padrão de idade e frequências relativas e absolutas de dados referentes ao gênero de indivíduos adultos do Centro universitário AGES, localizado no município de Paripiranga-BA, 2018.

\begin{tabular}{ll}
\hline Variáveis & $\mathbf{X}(\mathrm{DP})$ \\
\hline Idade (anos) & $24,42(6,41)$ \\
Gênero & $\mathbf{N}(\%)$ \\
Feminino & $36(36 \%)$ \\
Masculino & $64(64 \%)$
\end{tabular}

Fonte: Dados coletados pelos autores, 2018.

O setor industrial brasileiro viu a necessidade de uma maior produção de lanches e comidas mais rápidas, frente a mudanças do crescimento das despesas com alimentação fora de casa como em restaurantes tipo fastfood, alimentação no ambiente de trabalho, escolar ou universitário. Esses alimentos que facilitavam o consumo alimentar dos indivíduos, também começaram a ser considerados mais prazerosos, menos perecíveis e que poderiam ser consumidos em qualquer ambiente. (CORREA BA, 2016 e MONTEIRO CA, et al., 2010).

Atualmente, o consumo de fastfoods é um quadro comum observado em todas as faixas etárias. Com necessidade de praticidade e rapidez da vida moderna, a incidência de maus hábitos alimentares tem crescido consideravelmente, principalmente no público adulto. É comum vê-los se alimentando em lanchonetes e cantinas onde o cardápio costuma ser repleto de alimentos industrializados ricos em gordura e bebidas açucaradas, como refrigerantes e sucos artificiais (BASTOS TPF, et al., 2014; FERREIRA TS, et al., 2012).

Se tratando da população de universitários brasileiros, foi observado que os mesmos, não seguem na maioria das vezes, hábitos alimentares saudáveis e que há uma maior prevalência do consumo de alimentos industrializados ricos em gorduras e açucares e um baixo consumo de hortaliças e frutas (CORREA BA, 2016; CANSIAN ACC, et al., 2012; MENDES MLM, et al., 2016).

Tendo em vista que o presente estudo analisou os hábitos alimentares de universitários, um dos principais achados foi o alto consumo de industrializados e a quantidade excessiva de refrigerante consumida. Cerca de $43 \%$ dos participantes da amostra que declararam consumir refrigerante, alegaram que consomem mais de um litro por dia, o que pode ser um potencial risco a saúde (OLIVEIRA JS, et al., 2017).

A Tabela 2 ilustra a porcentagem do consumo de refrigerantes e industrializados, bem como outros hábitos alimentares dos universitários. Neste sentido, a ingestão dessas bebidas é comum em maior parte da amostra 
(55\%). Apesar de não ser tão frequente, maior parte dos indivíduos (77\%) consome alimentos industrializados. Quando questionados sobre a leitura de rótulos, a maioria observa o que está contido lá, mas muitos não compreendem o que significam as informações (Tabela 2).

Tabela 2 - Frequências relativas e absolutas da ingestão de refrigerantes, bebidas e alimentos industrializados por universitários adultos do Centro universitário AGES, localizado no município de Paripiranga-BA, 2018.

\begin{tabular}{|c|c|}
\hline \multicolumn{2}{|c|}{ Hábitos alimentares } \\
\hline Consumo de refrigerante & \\
\hline Sim & $55(55 \%)$ \\
\hline Não & 37 (37\%) \\
\hline Raro & $8(8 \%)$ \\
\hline \multicolumn{2}{|l|}{ Frequência de consumo } \\
\hline Todos os dias & $6(6 \%)$ \\
\hline De 4 a 6 vezes por semana & $6(6 \%)$ \\
\hline Em média 2 vezes & $12(12 \%)$ \\
\hline $1 \mathrm{vez}$ & $32(32 \%)$ \\
\hline Raro & 44 (44\%) \\
\hline \multicolumn{2}{|l|}{ Quantidade média de consumo } \\
\hline 1 copo & $29(29 \%)$ \\
\hline 1 latinha & $26(26 \%)$ \\
\hline 1 litro & $2(2 \%)$ \\
\hline Mais que 1 litro & $43(43 \%)$ \\
\hline \multicolumn{2}{|l|}{ Tipos de bebida consumidas } \\
\hline Refrigerantes & $12(12 \%)$ \\
\hline Sucos & $45(43 \%)$ \\
\hline Achocolatados & $14(14 \%)$ \\
\hline Sucos e achocolatados & $6(6 \%)$ \\
\hline Achocolatados e refrigerantes & $8(8 \%)$ \\
\hline Todas as opções & $0(0 \%)$ \\
\hline Raro & $15(15 \%)$ \\
\hline \multicolumn{2}{|l|}{ Consumos de industrializados } \\
\hline Sim & $77(77 \%)$ \\
\hline Não & $9(9 \%)$ \\
\hline Raro & $14(14 \%)$ \\
\hline \multicolumn{2}{|l|}{ Frequência semanal } \\
\hline Todos os dias & $13(13 \%)$ \\
\hline De 4 a 6 vezes por semana & $12(12 \%)$ \\
\hline Em média 2 vezes & $9(9 \%)$ \\
\hline $1 \mathrm{vez}$ & $2(2 \%)$ \\
\hline Raro & $14(14 \%)$ \\
\hline \multicolumn{2}{|l|}{ Quantidade média por dia } \\
\hline Menos de 1 pacote de biscoito/salgadinho & $46(46 \%)$ \\
\hline 1 pacote de biscoito/salgadinho & $29(29 \%)$ \\
\hline Mais de 1 pacote de biscoito/salgadinho & $9(9 \%)$ \\
\hline Raro & $16(16 \%)$ \\
\hline \multicolumn{2}{|l|}{ Leitura de rótulos } \\
\hline Sim & $53(53 \%)$ \\
\hline Não & 47 (47\%) \\
\hline \multicolumn{2}{|c|}{ Conhecimento das informações contidas nos rótulos } \\
\hline Sim & $47(47 \%)$ \\
\hline Não & $37(47 \%)$ \\
\hline Raro & $17(17 \%)$ \\
\hline
\end{tabular}

Fonte: Dados coletados pelos autores, 2018.

Em relação ao consumo de industrializados, grande parte dos estudantes (77\%) consomem industrializados, mesmo que em quantidade moderada. Mesmo que o consumo pela maioria não seja diário, ainda assim, é um fator de risco para a saúde. Considerando as consequências do consumo desses alimentos 
para a saúde dos indivíduos, o Guia Alimentar para a População Brasileira (BRASIL, 2014) recomenda que o consumo de açúcares e gorduras não ultrapasse uma porção diária, correspondente a 110kcal/dia. Também deve ser levado em consideração que o consumo exagerado de industrializados e refrigerantes acarretam diversos malefícios à saúde e são fatores de risco para doenças crônicas não transmissíveis (DCNT).

De acordo com Buzzo MA et al. (2014) há evidências recentes da associação entre dieta e desenvolvimento de diabetes tipo 2. Além disso, o consumo de alimentos com alto teor calórico, gorduras saturadas, sódio e açúcar pode desencadear doenças cardiovasculares que está correlacionada diretamente ao consumo de quantidades inadequadas de alimentos.

Entre os problemas que os refrigerantes e as bebidas açucaradas podem causar, podemos destacar a grande densidade energética, pois o consumo de produtos sob forma líquida tem efeitos fisiológicos diferentes em relação à forma sólida, em relação à líquida, a sensação de saciedade é menor, fazendo com que ocorra a ingestão dos alimentos calóricos de forma sólida resultando no consumo de mais calorias (BASTOS TPF, et al., 2014; ESTIMA CCP, et al., 2011).

A publicidade de tais produtos é sofisticada, apelativa e convincente. Isso os torna mais desejáveis. As estratégias de marketing desses produtos são, em alguns casos, baseadas em alegações de saúde, como dizer que determinado alimento é rico em algum micronutriente, mesmo que no geral esse alimento tenha baixa qualidade nutricional. Os produtos ultra processados rotulados como "light", "suplementados", "fortificados" ou outra forma associativa com algo saudável, geralmente são intrinsecamente insalubres. Daí se dá a importância de lermos e entendermos os rótulos (CORREA BA, 2016).

Apesar de $47 \%$ da amostra ter relatado que lê e entende os rótulos, esse interesse não deve estar relacionado somente com a data de validade. Mas principalmente com interesse em uma alimentação saudável. A compreensão da informação nutricional nos rótulos dos alimentos está associa-se principalmente ao conhecimento nutricional. Fatores como experiência, características sociais e demográficas, conhecimento, assim como a confiança nos fornecedores de mensagens podem influenciar o hábito da leitura dos rótulos que deve ser incentivado e orientado por profissionais (SOARES DJ, et al., 2016).

Apesar dos resultados negativos já citados, o estudo aponta que há preocupação dos estudantes na qualidade dos hábitos alimentares, quando $45 \%$ destes alegam que preferem sucos naturais que refrigerantes, $44 \%$ dos universitários relataram que só tomam refrigerante raramente e $53 \%$ responderam que leem os rótulos dos alimentos. Esse comportamento pode ter fundamentação na formação acadêmica haja vista que o ambiente educacional é um fator influenciador dos hábitos alimentares dos estudantes e, portanto, um ambiente importante de pesquisa do consumo alimentar dos alunos e um local propicio a intervenção (CORREA BA, 2016; BUSATO MA, et al., 2015).

Em suma, a alimentação adequada também tem como tributo, o direito de acesso à informação cientificamente comprovada sobre alimentação saudável, alimentos seguros e adequados, ratificando a necessidade de realizar ações que habilitem a população a fazer escolhas saudáveis (BRASIL, 2013).

Diante do exposto a alimentação saudável está ligada a diversos fatores, dentre eles, o número de refeições, qualidade do que se come e consumo de alimentos saudáveis de preferência in natura. Neste contexto vale ressaltar a necessidade e importância do consumo de alimentos ricos em cereais integrais, frutas e vegetais que que são a base para uma alimentação saudável e equilibrada e estão associados à diminuição de exposição ao risco para doenças crônicas (MENDES MLM, et al., 2016).

\section{CONSIDERAÇÕES FINAIS}

Com o este estudo foi possível perceber que o consumo de industrializados de forma geral ainda é crescente, principalmente no público adulto e universitário. A falta de leitura ou incompreensão de rótulos também é um fato importante, tendo em vista que a indústria brasileira busca mascarar os seus alimentos para que o consumidor não os compreenda corretamente, fazendo-se necessário a criticidade durante a compra do produto, bem como no momento das escolhas alimentares. 


\section{REFERÊNCIAS}

1. BARRETO ML. Esboços para um cenário das condições de saúde da população brasileira 2022/2030. Fundação Oswaldo Cruz. Rio de Janeiro, vol. 2, p. 97-120, 2013.

2. BASTOS TPF, et al. Análise do perfil alimentar e do índice de sedentarismo e sobrepeso em estudantes universitários dos Cursos de Saúde. Revista Práxis. Ano VI, oㅜ 12. 2014.

3. BRASIL. Ministério da Saúde. Secretaria de Atenção à Saúde. Departamento de Atenção Básica. Política Nacional de Alimentação e Nutrição. Departamento de Atenção Básica. 1. ed., 1. Reimpr. - Brasília: Ministério da Saúde, 2013.

4. BRASIL. Ministério da Saúde. Secretaria de Atenção à Saúde. Guia alimentar para a população brasileira. 2ª Edição. Ministério da Saúde: Brasília, 2014.

5. BRASIL. Ministério da Saúde. Vigilância de Fatores de Risco e Proteção para Doenças Crônicas por Inquérito Telefônico: estimativas sobre frequência e distribuição sociodemográfica de fatores de risco e proteção para doenças crônicas nas capitais dos 26 estados brasileiros e no Distrito Federal em 2016. Ministério da Saúde, Secretaria de Vigilância em Saúde, Departamento de Vigilância de Doenças e Agravos não Transmissíveis e Promoção da Saúde. Brasília: Ministério da Saúde, 2017.

6. BUSATO MA, et al. Ambiente e alimentação saudável: percepções e práticas de estudantes universitários. Semina: Ciências Biológicas e da Saúde, Londrina, v. 36, n. 2, p. 75-84, jul./dez. 2015.

7. BUZZO ML, et al. Elevados teores de sódio em alimentos industrializados consumidos pela população brasileira. Rev. Inst. Adolfo Lutz; 73(1):32-9, 2014.

8. CASSIAN ACC, et al. Ingestão de frutas e hortaliças. Nutrire. São Paulo, v. 37 n. 1 p. 54- 63, 2012.

9. CORREIA BA. Determinantes do consumo de alimentos processados e ultra processados em estudantes da Universidade de Brasília (UnB), Distrito Federal. Dissertação (Trabalho de Conclusão de Curso). Departamento de graduação em Nutrição da Universidade de Brasília (UnB). Brasília, p. 61, 2016.

10. ENES CC, SLATER B. Obesidade na adolescência e seus principais fatores determinantes. Revista Brasileira de Epidemiologia. São Paulo, v.13(1): 163-71, 2010.

11. ESTIMA CCP, et al. Consumo de bebidas e refrigerantes por adolescentes de uma escola pública. Revista Paulista de Pediatria. v.29, n.1, p.41-45, São Paulo, 2011.

12. FERREIRA SR. Alimentação, nutrição e saúde: avanços e conflitos da modernidade. Revista ciência e cultura. Volume 62, no 4. São Paulo, outubro de 2010.

13. FERREIRA TS, et al. Obesidade central em jovens. Revista Science in Health. v.3, n.2, p. 61-73, 2012.

14. OLIVEIRA JS, et al. Avaliação do perfil sociodemográfico, nutricional e alimentar de estudantes de nutrição de uma universidade pública em Lagarto-SE. RASBRAN - Revista da Associação Brasileira de Nutrição. São Paulo, SP, Ano 8, n. 2, p. 37-42, Jul-Dez. 2017.

15. MENDES MLM, et al. Hábitos alimentares e atividade física de universitários da área de saúde do município de Petrolina-PE. Tempus, actas de saúde colet. Brasília, 10(2), 205-217, jun., 2016.

16. MONTEIRO CA, et al. A new classification of foods based on the extent and purpose of their processing. Cad. Saúde Pública, Rio de Janeiro, v. 26, n. 11, p. 2039-2049, 2010.

17. ORLANDO R, et al. Avaliação da alimentação e sua relação com as doenças crônicas não transmissíveis de um grupo de idosos de um município da região norte do estado do RS. Revista de Enfermagem. v. 6-7 n. 6-7 p. 203 217 2010-2011

18. PINHO PM, et al. Síndrome metabólica e sua relação com escores de risco cardiovascular em adultos com doenças crônicas não transmissíveis. Rev. Soc. Bras. Clín. Méd. v. 12, n. 1, p. 22-30, 2014.

19. SOARES DJ, et al. Análise do comportamento dos consumidores com relação à compreensão e entendimento das informações dos rótulos de alimentos. Revista AGROTEC - v. 37, n. 1, p. 105-111, 2016. 